- Prosthetic rehabilitation is often part of the corrective therapy in cases of advanced periodontal disease.

- Although implant treatment is sometimes indicated and successfully applied in such cases, it may not always be the treatment of choice.

- Cross-arch bridges have a record of low complications and can serve this group of patients well.

- The application of alternative materials to gold, such as cobalt/chromium, is associated with favourable biomechanical properties and reduced production costs.

\title{
Restoration of periodontally compromised dentitions using cross-arch bridges. Principles of perio-prosthetic patient management
}

\author{
S. Kourkouta, ${ }^{1}$ K. W. Hemmings ${ }^{2}$ and L. Laurell ${ }^{3}$
}

\begin{abstract}
Severe periodontal disease often leads to tooth loss, necessitating prosthetic rehabilitation to restore function and aesthetics. The concept of perio-prosthetic treatment using extensive bridges of cross-arch design was introduced approximately 30 years ago. Long term follow-up studies have shown that teeth with reduced periodontal support can be used as abutments for extensive fixed prostheses, provided periodontal disease has been treated successfully, and an effective recall programme has been instituted to prevent periodontal disease recurrence. Low complication rates have been reported with these extensive constructions.
\end{abstract}

This paper presents established principles of perio-prosthetic patient management. A clinical case is presented and discussed, to illustrate this treatment concept.

\section{INTRODUCTION: HISTORIC PERSPECTIVE}

Advanced periodontal disease is often associated with severe loss of tooth support and loss of teeth. Hopeless teeth have to be extracted as part of the initial (cause-related) therapy, whereas teeth with questionable prognosis that have not responded to the initial phase of periodontal therapy may have to be extracted following re-examination.

\footnotetext{
${ }^{1 *}$ Honorary Consultant, ${ }^{2}$ Consultant in Restorative Dentistry, Conservation Department, Eastman Dental Hospital \&t Institute, 256 Gray's Inn Road, University College London Hospitals NHS Foundation Trust, London; ${ }^{3}$ Senior Lecturer/Honorary Consultant in Periodontology, UCL Eastman Dental Institute, 256 Gray's Inn Road, London

${ }^{*}$ Correspondence to: Miss Styliani Kourkouta

Email:styliani.kourkouta@uclh.org
}

\section{Refereed Paper}

Accepted 14 December 2006

DOI: $10.1038 /$ bdj.2007.727

${ }^{\circ}$ British Dental Journal 2007; 203: 189-195
Prosthetic replacement is often necessary as part of the corrective therapy to restore function and aesthetics in the periodontally compromised dentition. The remaining teeth are often mobile and may require splinting to enhance patient comfort. Although such teeth cannot function individually, due to the severe loss of periodontal support, once splinted they can survive for a considerable time provided the periodontal infection is under control., ${ }^{1,2}$ Fixed bridges of a cross-arch design provide a degree of rigidity and result in a more favourable distribution of the masticatory load along the entire arch, rather than on individual units, therefore preventing overloading of abutment teeth with reduced periodontal support. ${ }^{3}$

Ante's law (1926) ${ }^{4}$ is generally referred to as safe prosthodontic design for bridges. It has largely influenced prosthodontic thinking and is still taught in undergraduate curricula around the world. It states that in planning for and designing fixed partial dentures, the pericemental area of the abutment teeth should be equal to or exceed that of the tooth or teeth to be replaced'. This concept has been questioned, since it attaches more importance to the number of teeth to be replaced than to the amount of remaining periodontal tissues supporting the abutments and thus the bridge constructions. ${ }^{5,6}$ Defiant to Ante's unproven postulation, extensive cross-arch bridges by far not fulfilling the prerequisites of Ante's law have been successfully provided since the 1970s as a means of rehabilitating periodontally compromised patients. Several long term follow-up studies ${ }^{1,6-9}$ have shown that fixed bridges can be placed and successfully maintained on a minimal number of abutment teeth with greatly reduced periodontal support, provided the prosthodontic treatment is: 1) preceded by adequate periodontal therapy, and 2) followed by a plaque control programme effective enough to prevent recurrence of periodontal disease. If presumptive abutments are well distributed and periodontal infection is under control, as little as $20-30 \%$ of the original periodontal 


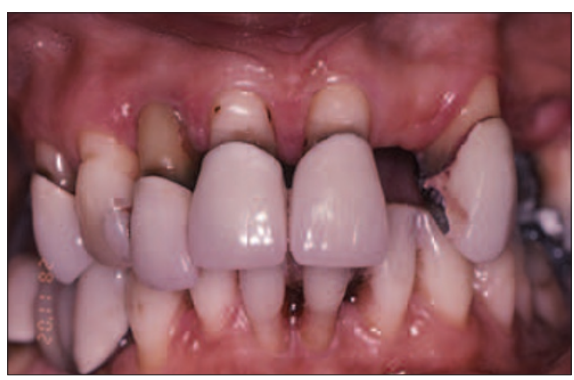

Fig. 1 Clinical presentation (plaque disclosing) following periodontal therapy

tissue support can be sufficient to carry fixed cross-arch bridges. ${ }^{6,9,10}$

\section{PRINCIPLES OF PERIO-PROSTHETIC TREATMENT}

Patient management and treatment sequence in perio-prosthetic therapy

As is true for any advanced restorative procedure, appropriate patient selection is critical. Direct patient involvement throughout the clinical stages of the treatment and during the maintenance phase is a prerequisite for a successful outcome. Patient compliance is of utmost importance; patients not only have to understand and appreciate what is required of them during treatment, they also need to take responsibility for their self-performed plaque control and overall care of the prosthesis.

The management of the perio-prosthetic patient consists of the following sequence: ${ }^{11}$

1) Baseline examination/diagnosis/ prognosis/patient motivation

2) Preliminary treatment plan/initial therapy

3) Re-examination after three to six months

4) Definitive treatment plan/corrective therapy:

i. Extraction of hopeless teeth and replacement with a temporary cross-arch bridge

ii. Periodontal surgery for pocket elimination and/or crown lengthening

iii.Supportive periodontal therapy for three to six months

iv. Reassessment

v. Provision of the final cross-arch bridge

5) Maintenance therapy (three to six month recall).

\section{Design of the perio-prosthesis}

It is important to visualise the final result before commencing treatment by

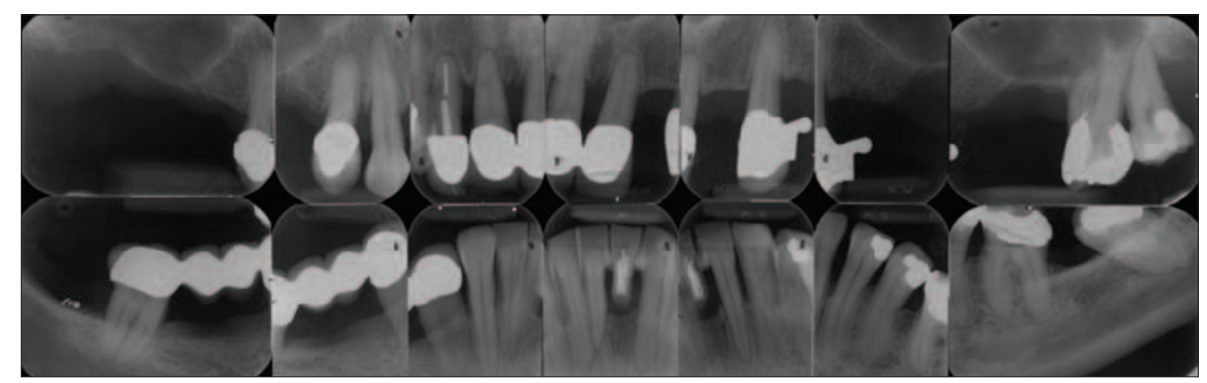

Fig. 2 Full mouth periapical radiographs after completion of active periodontal therapy; the 12 is deemed hopeless and scheduled for extraction

performing a diagnostic wax-up of the intended reconstruction on appropriately mounted diagnostic casts. A semi-adjustable articulator is normally adequate. The design of the prosthesis can thus be determined and a vacuum formed template produced to assist with construction of the provisional restoration at the abutment preparation visit.

\section{Number and distribution of abutment} teeth/cantilever extensions

A 10 or 12-unit bridge is usually aimed for, depending on functional status and aesthetic requirements, eg quantity and quality of potential abutment teeth and remaining periodontal support, occlusal contacts, display of posterior teeth. The number and distribution of abutment teeth are of primary importance. Although cross-arch bridges have been provided in the past, where only two canine teeth have served as abutments for 10 or even 12-unit prostheses with good long-term results, ${ }^{9,12,13}$ it is generally considered safer to have at least four or six abutment teeth for such constructions. Symmetrical distribution of the abutment teeth, eg a situation where maxillary central incisors, canines and second premolars are present and can serve as abutments for a 10 or 12-unit bridge, would be ideal to obtain optimal load distribution to the remaining periodontium. A minimum of 30\% remaining periodontal support of the abutment teeth is desirable. A maximum of two cantilever units may be incorporated uni- or bilaterally in cross-arch bridges, for functional and/or aesthetic reasons, provided certain prerequisites are fulfilled. ${ }^{9}$ Posterior extension with one or two premolars will ensure occlusal stability by preventing anterior tilting. It is preferable that distal abutment teeth adjacent to cantilever segments are not endodontically treated, to reduce the risk of abutment tooth fracture. ${ }^{14,15}$
If posts are present, a ferrule effect of at least $3 \mathrm{~mm}$ should be provided.

\section{Type of restoration margins}

Often the amount of attachment loss in these cases allows for supra-gingival placement of the restoration margins. This simplifies restorative procedures and facilitates plaque control at the critical restoration-tooth interface by the patient, as well as improved monitoring conditions for the operator during the maintenance phase. Shallow chamfer or even feather-edge preparation margins are more appropriate, since the preparations extend onto the root surfaces and a shoulder or heavy chamfer would result in pulp exposure. The type of preparation dictates provision of metal collars in the majority of cases. However, this is usually not an aesthetic concern, as the margins are normally covered by the soft tissues of the lips and cheeks and there is no obvious display of metal, unless the patient has an extremely high smile line.

\section{Biomechanical considerations}

The biomechanical factors that affect long-term prognosis of these extensive reconstructions have been defined by Laurell et al. ${ }^{9}$ and Lundgren and Laurell ${ }^{16}$ as:

\section{Retention}

The presence of long clinical crowns in periodontal dentitions, as a sequela of clinical attachment loss and/or pocket elimination periodontal surgery, is favourable in terms of retention and resistance form for the fixed prosthetic reconstruction. Optimal retention is secured by almost parallel preparations of the abutment teeth. Clinical experience dictates that the most difficult surfaces to parallel are the distal walls of the posterior abutments in relation to the labial surfaces of the anterior 
teeth. Surveying of intermediate casts is often indicated to ensure appropriate tooth reduction.

\section{Dimension}

The recommended dimensions for the metal framework, at least mesial and distal to the distal retainer crowns, are $5 \mathrm{~mm}$ in height by $4 \mathrm{~mm}$ in width. These dimensions should preferably be observed along the entire construction, to avoid fatigue and material fracture.

\section{Occlusion}

Even contacts should be established anteriorly as well as posteriorly, with freedom in centric occlusion. The occlusal morphology should guide the occlusal forces in an axial direction. The palatal surfaces of the maxillary anterior teeth are given a functional morphology ${ }^{17}$ to ensure axial load direction. Overbite and overjet should be minimal and the steepness of cuspal inclines reduced. Lateral movements should be anterior guided with no contacts on the cantilevers. The occlusal contacts should be monitored regularly and adjusted accordingly during maintenance, as there is a tendency for greater functional wear anteriorly, which might result in premature contacts on the cantilever segments of the bridgework. ${ }^{9,18,19}$

\section{Occlusal load and chewing efficiency}

The magnitude and distribution of occlusal load on cross-arch bridges of different designs (end-abutment, uni- or bilateral cantilever) have been studied extensively by applying a method that used several strain-gauge transducers mounted into artificial crowns, bridge pontics or removable dentures. The method allowed measurement of occlusal load in various parts of the dentition and over the entire dentition simultaneously during normal function. ${ }^{18,20}$ The total occlusal load as well as the distribution of the forces on the entire cross-arch bridge during chewing and jaw closing were studied. The results showed that the magnitude of occlusal load on the cross-arch bridges during normal function varied considerably between individuals, which is also in agreement with Carlson et al. ${ }^{21}$ The mean total chewing force on crossarch bridges of different designs was 55 to $121 \mathrm{~N}$, whereas the mean maximal bite force in 'habitual occlusion' varied from 264 to $320 \mathrm{~N}^{16}$
In the presence of bilateral end-abutments, the chewing and biting forces were significantly higher in the posterior than in the anterior regions. The load on the second cantilever unit(s) was considerably lower compared to the first when the bridge occluded with natural teeth, provided ideal occlusal contacts had been established. A minor $(80 \mu \mathrm{m})$ experimentally induced premature occlusal contact on the second cantilever unit, however, dramatically increased the load on the cantilever and thereby the stress to the bridge construction and distal abutment with overt risk of fractures. There was a general tendency towards lower chewing and biting forces as the amount of periodontal support of the abutment teeth decreased. The chewing ability of subjects with cross-arch bridges was almost as good as that of subjects with complete healthy dentitions. ${ }^{20}$ In their study of 11 'experimental' 12-unit cross-arch bridges supported by two mandibular canines, Carlson et al. ${ }^{21}$ confirmed that the masticatory efficiency improved after the prosthodontic treatment without any symptoms of dysfunction of the masticatory system.

\section{COMPLICATIONS}

Fixed prosthodontic treatment is associated with certain inherent risks, which can be of a biological or technical nature:

1) Biological

a) Caries

b) Endodontic complications

c) Periodontal disease progression

2) Technical

a) Loss of retention

b) Fracture of construction

c) Fracture of abutment teeth.

Biological complications can be prevented to an extent by regular maintenance visits and a high standard of self-performed plaque control. Endodontic complications are most likely related to the trauma induced by the restorative procedures and can be as high as 15\% on abutment teeth, as opposed to $3 \%$ loss of vitality of non-abutment teeth, as observed by Bergenholtz and Nyman ${ }^{22}$ in a retrospective study of 52 perioprosthetic patients 6 to 11 years after treatment.

The most common technical complication is loss of retention and that can be avoided by adhering to sound

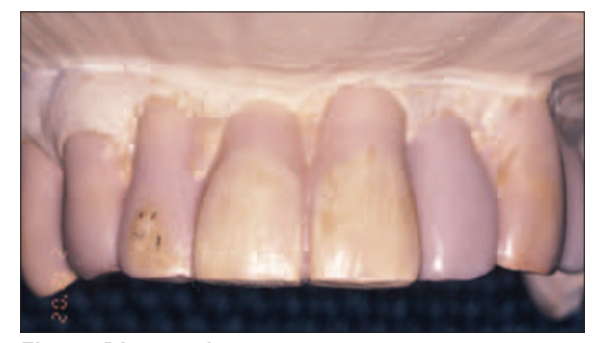

Fig. 3 Diagnostic wax-up 14-24

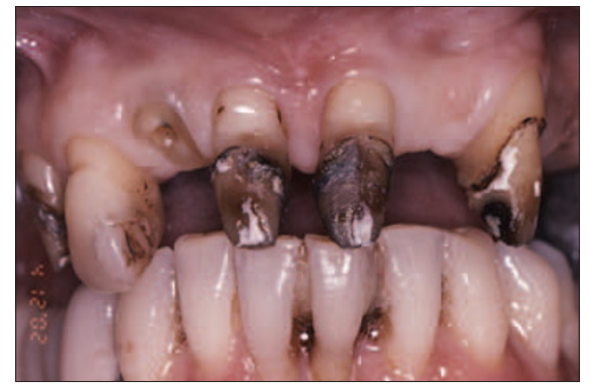

Fig. 4 Removal of old restorations

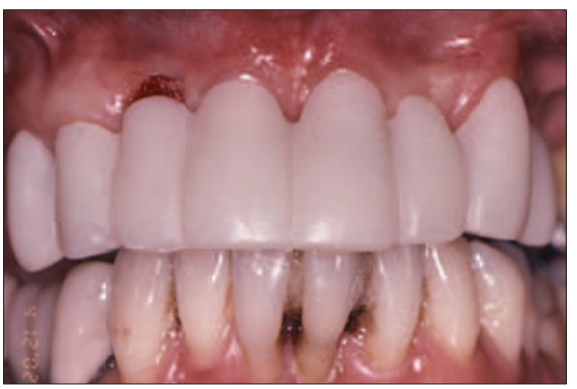

Fig. 5 Provision of temporary bridge

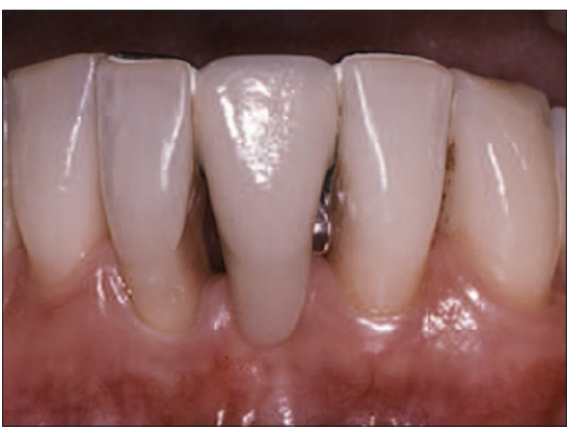

Fig. 6 Provision of fixed-fixed resin bonded bridge replacing the 31

principles of tooth preparation. ${ }^{1,19}$ Technical complications are relatively low with these bridges compared to other types of fixed prostheses. Nyman and Lindhe ${ }^{1}$ reported a total of less than $8 \%$ technical complications following an eight-year maintenance period of 332 bridges in 251 patients who had been treated for advanced periodontal disease. These consisted of loss of retention (3.3\%), fracture of abutment teeth $(2.4 \%)$ and fracture of the bridgework (2.1\%). Yi et al. ${ }^{6}$ evaluated a total of 43 cross-arch bridges in 34 patients, and concluded that $86 \%$ of the constructions 

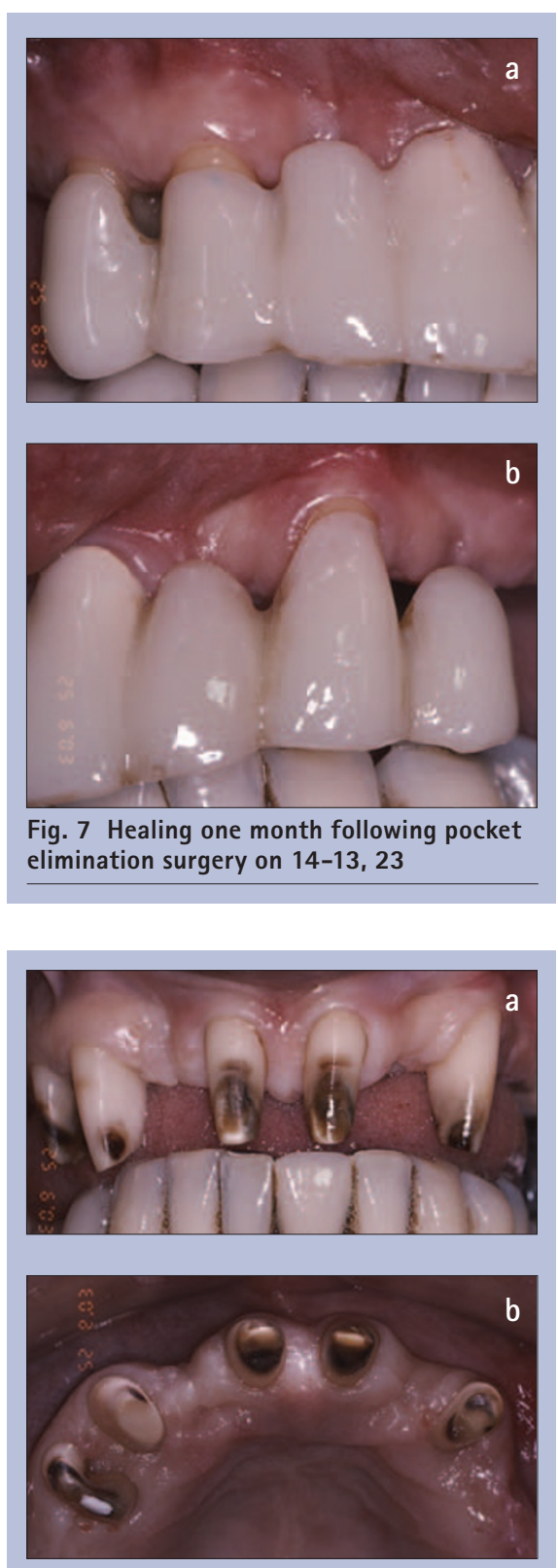

Fig. 8 Abutment preparation, front and occlusal view

were still in function after an average time of 15 years. The low rate of complications in these cases is attributed to the special characteristics of periodontal abutments, ie long clinical crowns that allow for increased retention and robust frameworks, as well as close follow-up of the patients to ensure high standards of plaque control and monitoring of the perio-prosthetic constructions on a regular basis. Overall, failures in this type of extensive fixed prostheses (i) increase with time, (ii) are more common in cantilever constructions, and (iii) occur more frequently in patients treated by general dental practitioners than at a specialist clinic. ${ }^{23}$

Based on the above, it can be stated that the indications and contra-indications for extensive tooth-supported fixed prosthodontic treatment in periodontally compromised patients are as follows:

\section{Indications}

- Mobility of the remaining periodontally treated teeth, affecting patient comfort and/or chewing ability

- A jaw relationship that permits establishment of anterior occlusal contacts

- Favourable distribution of potential abutment teeth

- Existing restorations in need of replacement

- Adverse conditions for implant treatment, eg medical reasons, lack of appropriate bone dimensions, proximity of anatomical structures (inferior alveolar nerve, maxillary sinus), financial considerations

- Patients' preference to maintain their own teeth.

\section{Contra-indications}

- Lack of patient motivation/compliance

- Unrealistic aesthetic demands; these constructions are mainly functional and, although excellent aesthetics can be achieved, this is not the primary goal of treatment

- A jaw relationship that does not permit establishment of anterior occlusal contacts, eg excessive overjet in Class II division 1 cases

- Unfavourable abutment distribution, resulting in excessive load over long pontic spans or unbounded cantilever segments

- Inadequate dental laboratory support

- Financial considerations.

\section{CASE PRESENTATION}

A case is presented of a 52-year-old female patient (Figs 1,2) to illustrate this perio-prosthetic treatment concept. The patient had periodontal therapy two years previously in the Periodontology Clinic, Eastman Dental Hospital (EDH) Et Institute, London. She required prosthetic rehabilitation, having lost a number of teeth due to severe periodontal disease as well as dental caries in the past. The remaining teeth were extensively restored. Perio-prosthetic treatment was carried out as part of a five-year prospective clinical case series study, which is currently in progress at EDH. The two-year results of that study have been presented elsewhere. ${ }^{24}$
Previous periodontal treatment consisted of: (i) initial periodontal therapy, (ii) re-examination, (iii) periodontal surgery, and (iv) supportive periodontal therapy. The patient was wearing a maxillary removable acrylic partial denture as a temporary prosthesis. Tooth 31 had been replaced at the time of initial periodontal therapy with an immediate adhesive resin bonded bridge using the crown of the tooth as a pontic. There were deficient restorations in need of replacement on:

(a) 11, 12, 14, 21, 23: porcelain-fusedto-metal crowns had been present on these teeth for over 20 years

(b) 13: extensive mesial Class III composite restoration.

\section{Medical history}

There was history of Hepatitis A 10 years previously.

\section{Diagnoses}

1) Acquired tooth loss (secondary to periodontal disease and caries)

2) Deficient restorations on 11, 12, 13, 14, 21, 23

3) Migration 12.

\section{Treatment plan/Treatment}

1) Diagnostic wax-up (Fig 3). Decision to proceed with a maxillary 10-unit cross-arch bridge ${ }^{15} 1413^{12} 1121^{22}$ $23{ }^{24} 25$ (The superscript font given here, which also appears in stages 3 and 7 , denotes the pontics and cantilevers)

2) Extraction of tooth 12 and provision of temporary bridge 14-24 (Figs 4, 5). This was performed in a oneday visit; the old restorations were removed and the abutment teeth (14, 13, 11, 21, 23) prepared. The temporary bridge was constructed using glass fibre (EverStick ${ }^{\circledR}$ StickTech, Stick Tech Ltd 0y, P. 0. Box 114, Fin-20521 Turku, Finland) reinforced resin (IntegrityTM temporary crown and bridge material, Dentsply International Inc., Milford, DE 199630359, USA) in an indirect method

3) Provision of a fixed-fixed resin bonded bridge $41^{31} 32$ (Fig. 6)

4) Localised pocket elimination/ crown lengthening surgery 14-13, 23

5) Supportive periodontal therapy

6) Reassessment (Fig. 7)

7) Provision of the final cross-arch bridge ${ }^{15} 1413^{12} 1121^{22} 23^{2425}$ 
(Figs 8-14): The preparations of the abutment teeth were refined following periodontal surgery (Fig. 8) and a working impression was made using heavy/light body addition cured silicone (President Coltène ${ }^{\circledR}$ Whaledent, Coltène/ Whaledent AG, Feldwiesenstrasse 20, 9450 Altstätten, Switzerland) in a custom acrylic tray.

The laboratory work was provided by Aurum Tandteknik AB, Örebro, Sweden. The framework was constructed using Wirobond ${ }^{\circledR}$ C (Bego, Bremen, Germany), a nickel-free cobalt/chromium $(\mathrm{Co} / \mathrm{Cr})$ alloy with veneering capacity. Metal try-in/jaw registration using Duralay resin (Duralay, Reliance, Dental Mfg. Co., IL 60482, USA) (Fig. 9) and porcelain try-in at bisque bake stage allowed refining of the occlusion. The final bridge (Figs 10-12) was cemented permanently using zinc phosphate cement (PhosphaCem ${ }^{\circledR}$ IC, Ivoclar Vivadent AG, FL-9494 Schaan, Liechtenstein)

8) Maintenance care at three to sixmonth intervals (Figs 15-16).

\section{DISCUSSION}

This paper describes the principles of rehabilitation of periodontally compromised patients using extensive bridges of cross-arch design. One of the sequelae of advanced periodontal disease is tooth loss. It is accepted that not every missing tooth has to be replaced, especially in posterior parts of the mouth, provided there are stable occlusal contacts, and the shortened dental arch concept is well established. ${ }^{25,26}$ However, the number and distribution of missing units, together with patient comfort factors, often render prosthetic rehabilitation necessary. Fixed restorations are generally considered preferable because they splint mobile teeth, resulting in a more favourable distribution of functional load to the remaining periodontium. ${ }^{3,27}$

The concept of perio-prosthetic treatment was introduced in Sweden in the 1970s. ${ }^{7,8}$ The provision of extensive cross-arch bridges in those days in cases that often seemed to be hopeless no doubt reflects not only the patients' preference for a fixed replacement of their missing teeth, but also the operators' conviction for the potential of these constructions. Implant treatment was not available as a treatment option at that time. However, it is possible that today a great number of periodontally involved teeth may be 'sacrificed' in preference to implant treatment in periodontally compromised patients. Although implants can be integrated successfully in the treatment of periodontally compromised dentitions, ${ }^{28-}$ ${ }^{32}$ a number of studies have raised concerns about long-term survival and success in cases with a history of periodontitis. ${ }^{33-36}$ In a 10-year study of the ITI $^{\circledR}$ dental implant system, Karoussis et al. ${ }^{37}$ reported lower survival and success rates and a higher percentage of biological complications for the group of patients who had lost their teeth due to periodontitis compared to patients without a history of periodontal disease. Certainly, periodontal disease should be under control prior to implant placement, to reduce the risk of peri-implantitis. ${ }^{38-40}$ A recent systematic review on the outcome of implant therapy in patients with previous tooth loss due to periodontitis ${ }^{41}$ concluded that, although implant survival did not differ in patients with and without a history of periodontitis, there was an increased incidence of periimplantitis and peri-implant marginal bone loss in the group of patients who had lost teeth due to past periodontal disease. It was suggested that appropriately designed long-term studies are required before any final conclusions can be drawn about the outcome of implant treatment in patients with a history of periodontitis.

In the presented case, extensive bone grafting would have been required for horizontal and vertical ridge augmentation prior to implant placement. Often patients are not willing to undergo extensive and potentially unpredictable bone grafting procedures, which have their own morbidity implications, especially when bone is harvested from extra-oral sites, ie the iliac crest. Financial and time factors are also crucial in determining the most appropriate treatment option for each patient.

The original cross-arch bridges were made using gold frameworks and acrylic veneers, mainly because these were the state-of-the-art materials in use in fixed prosthodontics at that time. In the mid-1980s, advances in the field of dental ceramics led to almost universal application of porcelain as the veneering material. Increases in the price of gold during the 1990s, and lately in

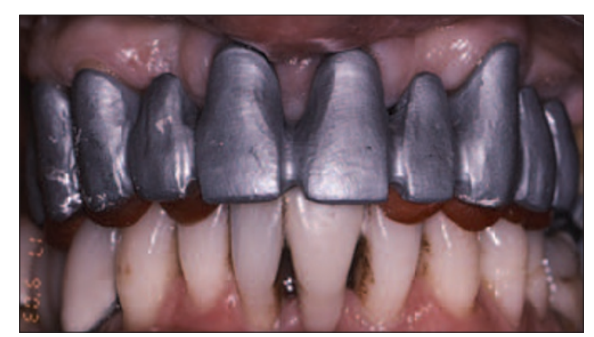

Fig. 9 Try-in of the metal framework and jaw registration using Duralay resin

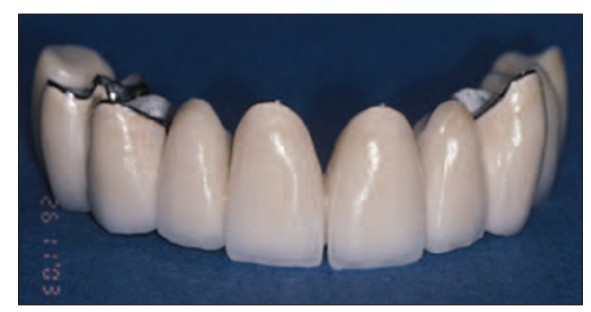

Fig. 10 Final bridge, showing the subtle metal collars on the retainers

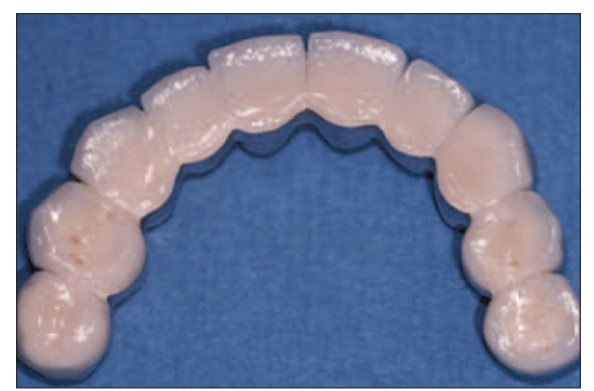

Fig. 11 Occlusal view of the final bridge showing the functional configuration of the occlusal table in the maxillary anterior region

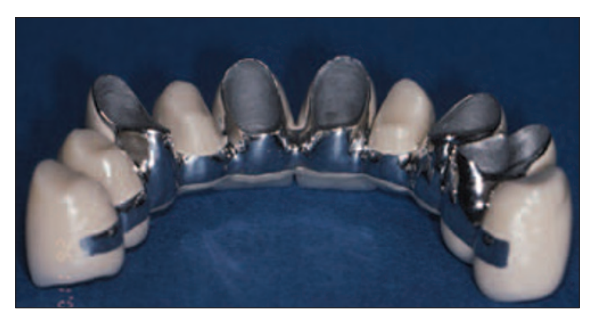

Fig. 12 Palatal view of the final bridge showing the extent of the metal framework

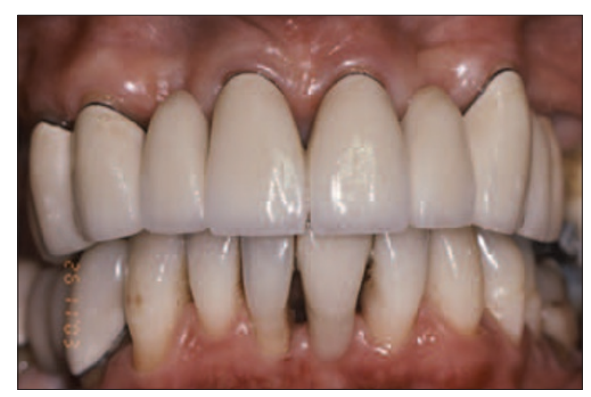

Fig. 13 Insertion of the final cross-arch bridge 15-25

Sweden, changes in the reimbursement regulations of dental care for the elderly $(65+)$ have moved the focus to non-precious alloys, as a more economic 
alternative to gold. ${ }^{42}$ The cobalt-chromium alloy Wirobond ${ }^{\circledR} \mathrm{C}$ has been in use for a number of years. This alloy shows some favourable properties, particularly applicable to the fabrication of metalceramic fixed prostheses, such as biocompatibility, high corrosion resistance, no nickel or beryllium content, rigidity and dimensional stability, prevention of deformation during ceramic firing and reliable bond strength with ceramics, high heat resistance and low thermal conductivity. ${ }^{43}$ The processing of $\mathrm{Co} / \mathrm{Cr}$ is generally considered more demanding compared to gold alloys, and this

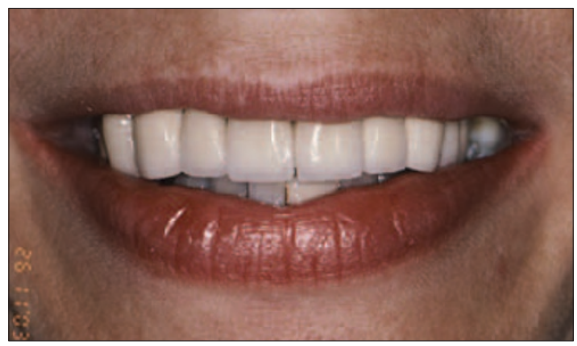

Fig. 14 Patient's smile

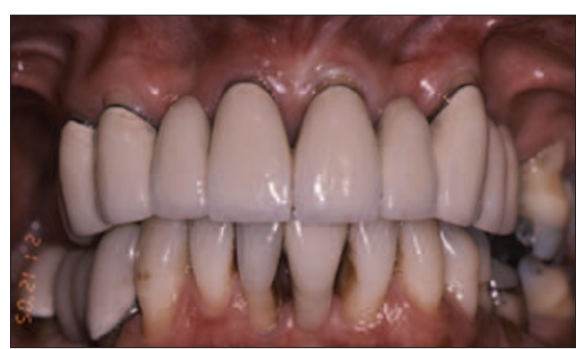

Fig. 15 Two-year review

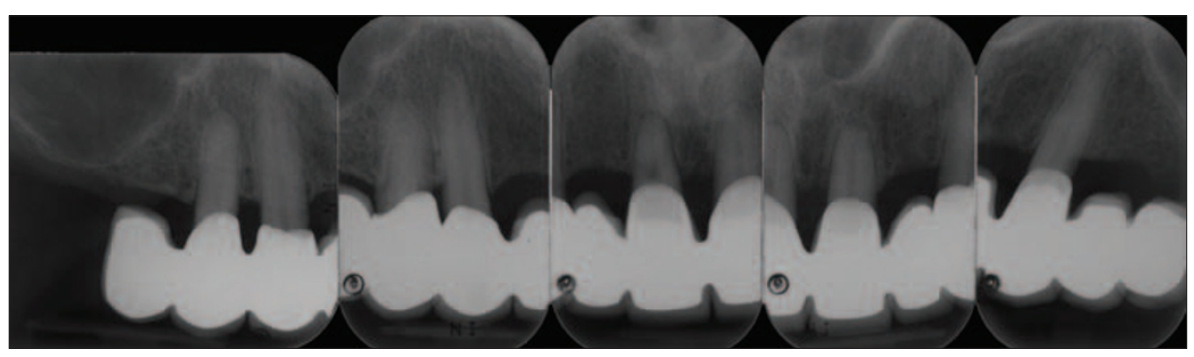

Fig. 16 Radiographic examination at three-year review

is where technical expertise becomes important. Overall, the cost reduction is considered to be approximately half the cost of bridge construction with high gold content alloys. ${ }^{42}$

The requirement to prepare intact teeth may be seen as a disadvantage in some of these perio-prosthetic cases. However, the alternative can be tooth loss in the long term. Restorative options should be carefully considered, and potential risks and benefits weighed before the most appropriate treatment plan is opted for. A number of the remaining teeth and potential abutments may be unrestored and, seemingly healthy, but they are periodontally compromised and that in itself influences the prognosis and decision making significantly.

Psychological issues stemming from tooth loss should not be underestimated. In the above presented case the patient felt this was 'miraculous' treatment and she was given 'a new lease of life'. Often patients who require perio-prosthetic treatment are middle aged, having to cope with a variety of psychological as well as newly discovered general health issues. Retaining a number of their own teeth as a foundation for a functional fixed prosthesis can be a great source of self confidence and improved outlook on life.

Considerable operator skill and expertise are required that can only be attained through appropriate training and personal reflection. In cases where treatment is provided by two specialists, ie a periodontist and prosthodontist working together, a high level of co-operation and team work is important during all stages of treatment. In addition, it has to be stressed that in conjunction with an individually tailored recall programme, patient compliance is crucial to a longterm successful outcome. This is both in terms of undergoing demanding and complex restorative treatment, but also being able to achieve and maintain an adequate level of self-performed plaque

control. In the words of Professor Sture Nyman, ${ }^{8}$ '...no treatment of this kind should be given to patients unwilling or unable to maintain a high standard of plaque control, or by dentists unwilling or unable to diagnose the presence of bacterial deposits on the tooth surfaces'.

1. Nyman S, Lindhe J. A longitudinal study of combined periodontal and prosthetic treatment of patients with advanced periodontal disease. $J$ Periodontol 1979; 50: 163-169.

2. Nyman S, Ericsson I. The capacity of reduced periodontal tissues to support fixed bridgework. J Clin Periodontol 1982; 9: 409-414.

3. Nyman S R, Lang N P. Tooth mobility and the biological rationale for splinting teeth. Periodontol
2000 1994; 4: 15-22

4. Ante I H. The fundamental principles of abutments. Thesis. Michigan State Dent Soc Bull 1926; 8: 14-23.

5. Laurell L, Lundgren D. Periodontal ligament areas and occlusal forces in dentitions restored with cross-arch bilateral end abutment bridges. J Clin Periodontol 1985; 12: 850-860.

6. Yi S W, Ericsson I, Carlsson G E et al. Long-term follow-up of cross-arch fixed partial dentures in patients with advanced periodontal destruction. Acta Odontol Scand 1995; 53: 242-248.

7. Lundgren D, Nyman S, Heijl L et al. Functional analysis of fixed bridges on abutment teeth with reduced periodontal support. J Oral Rehabil 1975; 2: 105-116.

8. Nyman S, Lindhe J, Lundgren D. The role of occlusion for the stability of fixed bridges in patients with reduced periodontal tissue support. J Clin Periodontol 1975; 2: 53-66.

9. Laurell L, Lundgren D, Falk H et al. Long-term prognosis of extensive polyunit cantilevered fixed partial dentures. J Prosthet Dent 1991; 66: 545-552.

10. Lundgren D. Prosthetic reconstruction of dentitions seriously compromised by periodontal disease. J Clin Periodontol 1991; 18: 390-395.

11. Lindhe J, Nyman S, Lang N P. Treatment planning. In Lindhe J, Karring T, Lang N P (Ed). Clinical periodontology and implant dentistry. $4^{\text {th }}$ edn. pp 414-431 (Chapter 19). Oxford: Blackwell Munksgaard, 2003

12. Carlson B R, Yontchev E, Carlsson G E. Extensive fixed partial dentures on mandibular canine teeth: a 5-year recall study. Int J Prosthodont 1989; 2: 265-271.

13. Öwall B E, Almfeldt I, Helbo M. Twenty-year experience with 12-unit fixed partial dentures supported by two abutments. Int J Prosthodont 1991; 4: 24-29.

14. Randow K, Glantz P O. On cantilever loading of vital and non-vital teeth. An experimental clinical study. Acta Odontol Scand 1986; 44: 271-277.

15. Randow K, Glantz P O, Zoger B. Technical failures and some related clinical complications in extensive fixed prosthodontics. An epidemiological study of long-term clinical quality. Acta Odontol Scand 1986; 44: 241-255.

16. Lundgren $D$, Laurell L. Biomechanical aspects of fixed bridgework supported by natural teeth and endosseous implants. Periodontol 2000 1994; 4: 23-40.

17. Beyron H. Occlusion: point of significance in planning restorative procedures. J Prosthet Dent 1973; 30: 641-652.

18. Laurell L, Lundgren D. Influence of occlusion on posterior cantilevers. J Prosthet Dent 1992; 67: 645-652.

19. Hämmerle C H F, Ungerer M C, Fantoni P et al. Long-term analysis of biologic and technical aspects of fixed partial dentures with cantilevers. Int J Prosthodont 2000; 13: 409-415.

20. Laurell L. Occlusal forces and chewing ability in dentitions with cross-arch bridges. Thesis. Swed Dent J, Supplement 26, Stockholm 1985.

21. Carlson B R, Carlsson G E, Helkimo E et al. Masticatory function in patients with extensive fixed cantilever prostheses. J Prosthet Dent 1992; 68: 918-923.

22. Bergenholtz G, Nyman S. Endodontic complications following periodontal and prosthetic treatment of patients with advanced periodontal disease. J Periodontol 1984; 55: 63-68.

23. Yi S W. Studies on oral rehabilitation of periodontally compromised dentitions. Thesis. Malmö University, Malmö 2001, Sweden.

24. Kourkouta S, Hemmings K, Petrie A et al. Cross-arch bridges made of porcelain-fused-toWirobond ${ }^{\circledR}$ C. Two-year clinical results. J Dent Res 2006: 85 (Special Issue B): Abstr 601.

25. Käyser A F. Limited treatment goals - shortened dental arches. Periodontol 2000 1994; 4: 7-14.

26. Kanno T, Carlsson G E. A review of the shortened dental arch concept focusing on the work by the Käyser/Nijmegen group. J Oral Rehabil 2006; 33: $850-862$. 
27. Hämmerle C H F. Success and failure of fixed bridgework. Periodontol 2000 1994; 4: 41-51.

28. Ellegaard B, Baelum V, Karring T. Implant therapy in periodontally compromised patients. Clin Oral Implants Res 1997; 8: 180-188.

29. Sbordone L, Barone A, Ciaglia R N et al. Longitudinal study of dental implants in a periodontally compromised population. J Periodontol 1999;

$$
\text { 70: 1322-1329. }
$$

30. Mengel R, Schröder T, Flores-de-Jacoby L. Osseointegrated implants in patients treated for generalized chronic periodontitis and generalized aggressive periodontitis: 3 - and 5-year results of a prospective long-term study. J Periodontol 2001; 72: 977-989.

31. Quirynen $M$, Peeters W, Naert I et al. Peri-implant health around screw-shaped c.p. titanium machined implants in partially edentulous patients with or without ongoing periodontitis. Clin Oral Implants Res 2001; 12: 589-594.

32. Baelum V, Ellegaard B. Implant survival in periodontally compromised patients. J Periodontol 2004; 75: 1404-1412.
33. Hardt C R E, Gröndahl K, Lekholm U et al. Outcome of implant therapy in relation to experienced loss of periodontal bone support. Clin Oral Implants Res 2002; 13: 488-494.

34. Evian C I, Emling R, Rosenberg E S et al. Retrospective analysis of implant survival and the influence of periodontal disease and immediate placement on long-term results. Int J Oral Maxillofac Implants 2004; 19: 393-398.

35. Rosenberg E S, Cho S C, Elian N et al. A comparison of characteristics of implant failure and survival in periodontally compromised and periodontally healthy patients: a clinical report. Int J Oral Maxillofac Implants 2004; 19: 873-879.

36. Van der Weijden G A, van Bemmel K M, Renvert S. Implant therapy in partially edentulous, periodontally compromised patients: a review. J Clin Periodontol 2005; 32: 506-511.

37. Karoussis I K, Salvi G E, Heitz-Mayfield L J A et al. Long-term implant prognosis in patients with and without a history of chronic periodontitis: a 10year prospective cohort study of the ITI ${ }^{\circledR}$ dental implant system. Clin Oral Implants Res 2003:
14: 329-339

38. Van Winkelhoff A J Goené $R$ J Benschop $C$ et al. Early colonization of dental implants by putative periodontal pathogens in partially edentulous patients. Clin Oral Implants Res 2000; 11: 511-520.

39. Van Winkelhoff A J, Wolf J W A. Actinobacillus actinomycetemcomitans-associated peri-implantitis in an edentulous patient. A case report. J Clin Periodontol 2000; 27: 531-535.

40. Sumida S, Ishihara K, Kishi M et al. Transmission of periodontal disease-associated bacteria from teeth to osseointegrated implant regions. Int J Oral Maxillofac Implants 2002; 17: 696-702.

41. Schou S, Holmstrup P, Worthington H V et al. Outcome of implant therapy in patients with previous tooth loss due to periodontitis. Clin Oral Implants Res 2006; 17 (Supplement 2): 104-123.

42. Bessing C. Cobalt-chromium alloys - excellent alternatives to high-gold alloys intended for metal ceramics. Tandläkartidningen 2003; 95: 38-43.

43. Strietzel R. Cobalt-chrome alloys with veneering capacity. BEGO document, Bremen 2001. 